\title{
A NOTE ON THE APPROXIMATION OF PDES WITH UNBOUNDED COEFFICIENTS - THE SPECIAL ONE-DIMENSIONAL CASE
}

\author{
F.F. Gonçalves ${ }^{1}$, M.R. Grossinho ${ }^{2} \S$, E. Morais ${ }^{3}$ \\ ${ }^{1}$ CEMAPRE-REM and ISEG, Universidade de Lisboa \\ Rua do Quelhas 6, 1200-781 Lisbon, PORTUGAL \\ Also with Universidade Europeia \\ Estrada da Correia 53, 1500-210 Lisbon, PORTUGAL \\ ${ }^{2}$ CEMAPRE-REM and ISEG, Universidade de Lisboa \\ Rua do Quelhas 6, 1200-781 Lisbon, PORTUGAL \\ ${ }^{3}$ CMAT, Universidade do Minho \\ Campus de Gualtar, 4710-057 Braga, PORTUGAL
}

\begin{abstract}
We consider the spatial approximation of the Cauchy problem for a linear uniformly parabolic PDE of second order, with nondivergent operator and unbounded time- and space-dependent coefficients, where equation's free term and initial data are also allowed to grow. We concentrate on the special case where the PDE has one dimension in space. As in [10], we consider a suitable variational framework and approximate the PDE problem's generalised solution in the spatial variable, with the use of finite-difference methods, but we obtain, for this case, consistency and convergence results sharper than the corresponding results obtained in [10] for the more general multidimensional case.
\end{abstract}

AMS Subject Classification: 65M06, 65M12, 65M15

Key Words: Cauchy problem; parabolic PDEs; unbounded coefficients; nondivergent operators; weighted Sobolev spaces; finite-difference methods

Received: October 29, 2019

(c) 2020 Academic Publications

${ }^{\S}$ Correspondence author 


\section{Introduction}

In this paper, we consider a Cauchy problem for a parabolic partial differential equation with one spatial dimension and unbounded coefficients, and use finite-difference methods to approximate in space the corresponding generalised solution. By concentrating on the special case of one dimension in space, we can prove a convergence result sharper than the corresponding result in [10] for the more general multidimensional case.

More precisely, let $L$ be the nondivergent second-order partial differential operator with real coefficients

$$
L u(t, x)=a(t, x) \frac{\partial^{2} u}{\partial x^{2}}+b(t, x) \frac{\partial u}{\partial x}+c(t, x)
$$

in $[0, T] \times \mathbb{R}$, with $T \in(0, \infty)$ a constant, and consider the initial value problem

$$
\frac{\partial u}{\partial t}=L u+f \text { in }[0, T] \times \mathbb{R}, \quad u(0, x)=g(x) \text { on } \mathbb{R} .
$$

The real-valued functions $f$ and $g$ are allowed to have polynomial growth. As for the linear operator, the coefficient functions $a$ and $b$ can also grow, quadratically and linearly, respectively, and $\partial u / \partial t-L$ is supposed to be uniformly parabolic.

In our study, we use the variational approach adequately obtained for dimension one from the utilised in [10] for the approximation of the multidimensional version of problem (1). However, as referred above, by considering the special one-dimensional case we can prove a stronger convergence result. In particular, the same order of accuracy is obtained under regularity assumptions weaker than those required in [10].

Apart from the interest of this subject in PDE theory, linear parabolic PDE problems arise in Biology, Physics and Mathematical Finance. We are mainly motivated by the latter application, namely to a class of stochastic models aimed to determine the arbitrage-free price of non path-dependent options of European type (see, e.g., [15]).

It is well known that the study of option pricing model can be reduced, with the use of a Feynman-Kač type formula, to solving Cauchy problems for the second-order linear parabolic PDE.

In fact, let us consider a general version of the Black-Scholes stochastic model for an option with fixed exercise, with the dynamics of underlying asset price modelled by the SDE

$$
d S_{t}=S_{t}\left(\mu d t+\sigma d B_{t}\right)
$$

where $S, \mu$ and $\sigma$ are, respectively, the price, the appreciation rate, and the volatility of the underlying asset, $B_{t}$ a standard Brownian motion, and both $\mu$ and $\sigma$ are taken time- and space-dependent. 
This is equivalent, by using a Feynman-Kač type formula, to solving the Cauchy problem for the second-order linear parabolic PDE with unbounded coefficients

$$
\frac{\partial V}{\partial t}+\frac{1}{2} \sigma^{2}(t, S) S^{2} \frac{\partial^{2} V}{\partial S^{2}}+r(t) S \frac{\partial V}{\partial S}-r(t) V=0 \quad \text { in }[0, T] \times \mathbb{R}^{+}
$$

with terminal condition

$$
V(T, S)=\phi(S) \quad \text { on } \mathbb{R}^{+}
$$

where $V$ is the option value, $r$ the risk-free interest rate, and $\phi$ the pay-off function (see, e.g., [15]). Then, the option price can be approximated by using nonprobabilistic numerical techniques to approximate the solution of the parabolic PDE problem (2)-(3).

Problem (1) clearly contains the (homogeneous) problem (2)-(3). Moreover, as in (1) we let the initial data unspecified, the pay-off in (3) can be considered in a large class of functions. We note that instead of the terminal-value formulation arising in the financial model, we consider in problem (1) the more standard initial-value formulation (one problem can be transformed in the other by a simple change of the time variable).

As done in [10] for the multidimensional case, in this paper we tackle the challenge posed to the spatial approximation by the unboundedness of the PDE coefficients assuming that the PDE does not degenerate. Due to this assumption, this work is not directly applicable to the financial problem which motivates us, and should be viewed instead as a step in that direction. The rate of convergence of the discretised problem's generalised solution to the exact problem's generalised solution is estimated. In order to facilitate the approach in space, we make use of basic one-step finite-difference schemes. For a study concerning the time approximation, we refer to $[9,18]$ where the discretisation of a general evolution equation problem of parabolic type can be found.

Note that if the procedure for obtaining implementable numerical schemes for problem (1) includes, as a first step, the problem's localisation to a bounded domain (see, e.g., $[1,2,17,20,19]$ ), the unboundedness of the PDE coefficients does not have to dealt with, as the PDE coefficients are bounded in the truncated domain.

But, if instead the problem is (semi) discretised in the whole space, and then the discretised problem is localised to a bounded domain, by imposing discrete artificial boundary conditions (see, e.g., $[4,5,6]$ ), the coefficient unboundedness remains a problem which must be tackled. As is [10], our study is meaningful in this latter case. 
Note also that most studies concerning the approximation of PDE problems in Finance consider the particular case where the PDE coefficients are constant (see, e.g., $[2,20]$ ). This occurs in Black-Scholes models (in one or several dimensions), when the underlying asset appreciation rate and volatility are taken constant: the simpler PDE with constant coefficients is obtained after a standard change of variables (see, e.g., [15] for the one-dimensional case, and [8] for the multidimensional case). When the drift and volatility are taken bounded functions of time and space, the above change of variables leads to a PDE with bounded coefficients (see, e.g., [1]). However, this procedure is not available under the weak regularity assumptions we consider.

We stress that finite-difference methods are particularly suitable for PDE problems related to Finance as most of them are Cauchy problems (therefore not showing the complex domain geometries which are one main reason to favour other numerical methods, e.g., finite-element methods). Also, although the theory concerning the finite-difference approximation of PDEs could be considered reasonably complete since some decades ago ${ }^{1}$, some important research is still ongoing (see, e.g., the recent studies $[12,13,14]$ ).

We summarise the content of this paper. Section 2 merely presents the particularisation of the framework utilised in [10] to the one dimensional case. We state well-known facts on the solvability of problem (1), and introduce a suitable class of weighted Sobolev spaces. Then, we discretise in space problem (1), with the use of a finite-difference scheme. We introduce discrete versions of the weighted Sobolev spaces, set a discrete framework, and state the existence and uniqueness of the discretised problem's generalised solution. In Section 3, we prove that this discrete generalised solution approximates the exact generalised solution, and determine the rate of convergence. In Section 4, we give an example, and study its approximation under the framework used in the article. Some final comments are made in Section 5 .

\section{The PDE problem and its discretisation}

We begin by reviewing a classical result on the solvability of the PDE problem under study in a variational framework. The fact that the coefficients of the linear operator are unbounded will be dealt with by using the so-called well-weighted Sobolev spaces ${ }^{2}$. Both the framework and the solvability result

\footnotetext{
${ }^{1}$ We refer to [21] for a brief summary of the method's history. For the application of the finite-difference method to option pricing, we refer to the review paper [3].

${ }^{2}$ See [11] for a comprehensive description of these spaces.
} 
considered in [10] for the multidimensional case are now particularised for the case of one dimension and presented for the reader's convenience.

Let $L$ be the second-order partial differential operator

$$
L=a(t, x) \frac{\partial^{2}}{\partial x^{2}}+b(t, x) \frac{\partial}{\partial x}+c(t, x)
$$

with real coefficients, defined in $Q=[0, T] \times \mathbb{R}$, with $T \in(0, \infty)$, and consider the PDE problem

$$
u_{t}=L u+f \text { in } Q, \quad u(0, x)=g(x) \text { on } \mathbb{R} .
$$

The functions $f$ and $g$ and the coefficients $a$ and $b$ are allowed to grow, and the operator $\partial u / \partial t-L$ is uniformly parabolic, according to the assumptions that will be stated later.

We introduce the well-weighted Sobolev spaces.

Let $U \subset \mathbb{R}$ be an open set, and $r$ and $\rho$ two positive smooth functions defined in $U$. Take an integer $m \geq 0$ and consider the weighted Sobolev space $W^{m, 2}(U ; r, \rho)$, the Banach space of all locally integrable functions $v: U \rightarrow \mathbb{R}$ such that for each integer $\alpha$, with $0 \leq \alpha \leq m$, the weak derivative $D^{\alpha} v$ exists, and

$$
\|v\|_{W^{m, 2}(U ; r, \rho)}:=\left(\sum_{\alpha \leq m} \int_{U} r^{2}\left|\rho^{\alpha} D^{\alpha} v\right|^{2} d x\right)^{1 / 2}<\infty .
$$

The above norm can be derived from the inner product

$$
(v, w)_{W^{m, 2}(U ; r, \rho)}:=\sum_{\alpha \leq m} \int_{U} r^{2} \rho^{2 \alpha} D^{\alpha} v D^{\alpha} w d x .
$$

Equipped with this inner product, $W^{m, 2}(U ; r, \rho)$ is a Hilbert space.

Notation. In the sequel, we omit the functions $r$ and $\rho$ from function space notation without risk of ambiguity. Also, when $U=\mathbb{R}$ the set is dropped from the notation. Finally, we will use the short notation $\|\cdot\|_{m, 2}$ for $\|\cdot\|_{W^{m, 2}(\mathbb{R} ; r, \rho)}$.

Consider the following assumptions on the functions $r$ and $\rho$ (see [11]).

Assumption 1. Let $m \geq 0$ be an integer, and $r>0, \rho>0$ smooth functions on $\mathbb{R}$. There exists a constant $K$ such that

1. $\left|D^{\alpha} \rho\right| \leq K \rho^{1-\alpha}$ for all $\alpha$ satisfying $\alpha \leq m-1$ if $m \geq 2$;

2. $\left|D^{\alpha} r\right| \leq K \frac{r}{\rho^{\alpha}}$ for all $\alpha$ satisfying $\alpha \leq m$; 
3. $\sup _{|x-y|<\varepsilon}\left(\frac{r(x)}{r(y)}+\frac{\rho(x)}{\rho(y)}\right)=K$ for some $\varepsilon>0, x, y \in \mathbb{R}$.

Remark 1. Note that in (1) in Assumption 1 nothing is required if $m<2$.

Remark 2. The set $\{(r, \rho)\}$ satisfying (1) - (3) in Assumption 1 is not empty $^{34}$. An interesting example, which we will use in a later section, includes the functions

$$
r(x)=\left(1+|x|^{2}\right)^{\beta}, \beta \in \mathbb{R}, \quad \text { and } \quad \rho(x)=\left(1+|x|^{2}\right)^{\gamma}, \gamma \leq \frac{1}{2} .
$$

We recall that a function $w: Q \rightarrow \mathbb{R}$ can be seen as a mapping from $[0, T]$ into a functional space by defining $(w(t))(x):=w(t, x)$. In the sequel we will take this point of view.

Next, we introduce some coercivity and regularity assumptions (see [11]).

Assumption 2. Let $r$ and $\rho$ be positive smooth functions on $\mathbb{R}$, and $m \geq 0$ an integer.

1. There exists a constant $\lambda>0$ such that $a(t, x) \geq \lambda \rho^{2}(x)$ for all $t \geq 0$, $x \in \mathbb{R}$;

2. The coefficient functions $a, b$, and $c$ of the operator $L$ and their derivatives in $x$ up to the order $m$ are measurable functions in $[0, T] \times \mathbb{R}$. Moreover, denoting by $D_{x}^{\alpha}$ the $\alpha^{t h}$ partial derivative operator with respect to $x$, there exists a constant $K$ such that

$$
\begin{gathered}
\left|D_{x}^{\alpha} a\right| \leq K \rho^{2-\alpha} \quad \forall \alpha \leq m \vee 1,\left|D_{x}^{\alpha} b\right| \leq K \rho^{1-\alpha}, \\
\left|D_{x}^{\alpha} c\right| \leq K \quad \forall \alpha \leq m,
\end{gathered}
$$

for all $t \in[0, T], x \in \mathbb{R}$;

3. $f \in L^{2}\left([0, T] ; W^{m-1,2}\right)$ and $g \in W^{m, 2}$.

Notation. For $m=0$ we use the notation $W^{-1,2}:=\left(W^{1,2}\right)^{*}$, where $\left(W^{1,2}\right)^{*}$ is the dual of $W^{1,2}$.

\footnotetext{
${ }^{3}$ See [11], citing O. G. Purtukhia, for a list of examples.

${ }^{4}$ The weight function considered in [1] for the variational formulation of the Black-Scholes equation clearly does not satisfy (1) - (2) of Assumption 1. We will discuss the suitability of this function in a future approach of the degenerate case of PDE problem.
} 
Then, we define the generalised solution of problem (4) (see [11]).

Definition 1. We say that $u \in C\left([0, T] ; W^{0,2}\right) \cap L^{2}\left([0, T] ; W^{1,2}\right)$ is a generalised solution of problem (4) if, for every $t \in[0, T]$ and all $\varphi \in C_{0}^{\infty}$,

$$
\begin{aligned}
(u(t), \varphi)=(g, \varphi)+\int_{0}^{t}\{ & -\left(a(s) D_{x} u(s), D_{x} \varphi\right) \\
& +\left(b(s) D_{x} u(s)-D_{x} a(s) D_{x} u(s), \varphi\right) \\
& +(c(s) u(s), \varphi)+\langle f(s), \varphi\rangle\} d s .
\end{aligned}
$$

Notation. The symbol $($,$) in the above definition denotes the inner$ product in $W^{0,2}$.

Finally, we state a well-known result concerning the existence and uniqueness of the solution of problem (4), and also its stability.

Theorem 2. Let $m \geq 0$ be an integer. Suppose that (1)-(2) in Assumption 1, with $m$ replaced by $m+1$, and (1)-(3) in Assumption 2 hold. Then problem (4) admits a unique generalised solution $u$ on $[0, T]$. Moreover

$$
u \in C\left([0, T] ; W^{m, 2}\right) \cap L^{2}\left([0, T] ; W^{m+1,2}\right)
$$

and the following estimate holds

$$
\begin{aligned}
\sup _{0 \leq t \leq T}\|u(t)\|_{m, 2}^{2}+\int_{0}^{T}\|u(t)\|_{m+1,2}^{2} d t & \\
& \leq N\left(\|g\|_{m, 2}^{2}+\int_{0}^{T}\|f(t)\|_{m-1,2}^{2} d t\right),
\end{aligned}
$$

with $N \in \mathbb{R}$ a constant.

The above statement can be obtained from a general result for evolution equations by using the suitable Gelfand triple of spaces (see, e.g., [11]).

Next, we discretise problem (4) in space. We set a suitable discrete framework by using a finite-difference scheme, and state an existence and uniqueness result for the discretised problem's generalised solution. The discrete setting parallels the corresponding one for the multidimensional case in [10], with the obvious modifications. We present it for sake of completeness, and also since 
the framework expressions become less heavy for the particular one dimensional problem we are studying.

Let

$$
Z_{h}=\{x \in \mathbb{R}: x=n h, \quad n=0, \pm 1, \pm 2, \ldots\},
$$

with $h \in(0,1]$, be the $h$-grid on $\mathbb{R}$.

For every $x \in Z_{h}$, consider the forward and backward difference quotients in space given, respectively, by

$$
\partial^{+} u=\partial^{+} u(t, x)=h^{-1}(u(t, x+h)-u(t, x))
$$

and

$$
\partial^{-} u=\partial^{-} u(t, x)=h^{-1}(u(t, x)-u(t, x-h)),
$$

Consider the discrete problem

$$
u_{t}=L_{h} u+f_{h} \text { in } Q(h), \quad u(0, x)=g_{h}(x) \text { on } Z_{h},
$$

where $L_{h}$ is the discrete operator

$$
L_{h}(t, x)=a(t, x) \partial^{-} \partial^{+}+b(t, x) \partial^{+}+c(t, x),
$$

$Q(h)=[0, T] \times Z_{h}$, with $T \in(0, \infty)$, and $f_{h}: Q(h) \rightarrow \mathbb{R}$ and $g_{h}: Z_{h} \rightarrow \mathbb{R}$.

We introduce the Banach spaces spaces $l^{0,2}(r)$ and $l^{1,2}(r, \rho)$, which are the discrete versions of the weighted Sobolev spaces of order 0 and 1 , respectively.

Consider functions $v: Z_{h} \rightarrow \mathbb{R}$ and the norm

$$
\|v\|_{l^{0,2}(r)}=\left(\sum_{x \in Z_{h}} r^{2}(x)|v(x)|^{2} h\right)^{1 / 2},
$$

The space $l^{0,2}(r)$ is defined as

$$
l^{0,2}(r):=\left\{v:\|v\|_{l^{0,2}(r)}<\infty\right\} .
$$

We observe that the above norm is induced by the inner product

$$
(v, w)_{l^{0,2}(r)}=\sum_{x \in Z_{h}} r^{2}(x) v(x) w(x) h,
$$

where $v, w \in l^{0,2}(r)$. Endowed with the inner product, the space $l^{0,2}$ is clearly a Hilbert space.

For functions $w: Z_{h} \rightarrow \mathbb{R}$, we introduce also the Banach space $l^{1,2}(r, \rho)$,

$$
l^{1,2}(r, \rho)=\left\{w:\|w\|_{l^{1,2}(r, \rho)}<\infty\right\}
$$

where

$$
\|w\|_{l^{1,2}(r, \rho)}^{2}=\|w\|_{l^{0,2}(r)}^{2}+\left\|\rho \partial^{+} w\right\|_{l^{0,2}(r)}^{2} .
$$


Notation. In the sequel, we omit the functions $r$ and $\rho$ from the notation for $l^{0,2}(r)$ and $l^{1,2}(r, \rho)$. Also, we will use the short notation $\|\cdot\| \|_{0,2}$ and $\|\cdot\|_{1,2}$ for $\|\cdot\|_{l^{0,2}(r)}$ and $\|\cdot\|_{l^{1,2}(r, \rho)}$, respectively.

As for the solvability study of the PDE problem (4), we will consider the functions $z: Q(h) \rightarrow \mathbb{R}$ as functions from $[0, T]$ into certain functional spaces defined by $(z(t))(x):=z(t, x)$ for all $t \in[0, T], x \in Z_{h}$. For these functions $z(t)$, we consider the space $C\left([0, T] ; l^{0,2}\right)$ of continuous $l^{0,2}$-valued functions on $[0, T]$ and the spaces

with $m=0,1$.

$$
L^{2}\left([0, T] ; l^{m, 2}\right)=\left\{z:[0, T] \rightarrow l^{m, 2}: \int_{0}^{T}\|z(t)\|_{m, 2}^{2} d t<\infty\right\},
$$

The data $f_{h}$ and $g_{h}$ in (8) will be assumed to satisfy certain conditions (see $[10])$.

Assumption 3. Let $r>0$ be a smooth function on $\mathbb{R}$. We assume that

1. $f_{h} \in L^{2}\left([0, T] ; l^{0,2}\right)$;

2. $g_{h} \in l^{0,2}$.

Remark 3. We observe that $\left|\partial^{+} a\right| \leq K \rho$ can be easily derived from (2) in Assumption 2 by using the mean value theorem.

We introduce the notion of generalised solution of problem (8) (see [10]).

Definition 3. We say that $u \in C\left([0, T] ; l^{0,2}\right) \cap L^{2}\left([0, T] ; l^{1,2}\right)$ is a generalised solution of (8) if, for every $t \in[0, T]$ and for all $\varphi \in l^{1,2}$,

$$
\begin{aligned}
(u(t), \varphi)=\left(g_{h}, \varphi\right)+\int_{0}^{t}\{ & -\left(a(s) \partial^{+} u(s), \partial^{+} \varphi\right) \\
& +\left(b(s) \partial^{+} u(s)-\partial^{+} a(s) \partial^{+} u(s), \varphi\right) \\
& \left.+(c(s) u(s), \varphi)+\left\langle f_{h}(s), \varphi\right\rangle\right\} d s .
\end{aligned}
$$

Notation. In the above definition, $($,$) denotes the inner product in l^{0,2}$.

Finally, we state an existence and uniqueness result for the solution of the discrete problem (8). The result also gives the stability of the approximation scheme. 
The theorem is proved in [10] for the more general multidimensional case; here, we just state its particularisation for the case of one dimension in space. The result is obtained in [10] by showing that the discretised problem is contained in the same general problem for evolution equations which the PDE problem can be cast into.

Theorem 4. Let (1)-(2) in Assumption 2 and (1)-(2) in Assumption 3 be satisfied. Then problem (8) has a unique generalised solution $u$ in $[0, T]$. Moreover,

$$
\sup _{0 \leq t \leq T}\|u(t)\|_{0,2}^{2}+\int_{0}^{T}\|u(t)\|_{1,2}^{2} d t \leq N\left(\left\|g_{h}\right\|_{0,2}^{2}+\int_{0}^{T}\left\|f_{h}(t)\right\|_{0,2}^{2} d t\right),
$$

where $N$ is a constant independent of $h$.

\section{Approximation results}

In this section, we are concerned with the consistency and convergence properties of the finite-difference scheme (8).

We emphasise that, by considering the special case of dimension one in space, we can prove a result concerning the convergence rate sharper than the corresponding result in [10] for the more general multidimensional case (see Remark 5 below), which stresses its interest.

We address firstly the consistency of the scheme, and prove that the difference quotients approximate the partial derivatives (with accuracy of order 1, as in the multidimensional case).

For that result, we assume that the weights $\rho$ are bounded from below by a positive constant. Notice that this amounts to assuming that the weights $\rho$ are increasing functions of $|x|$, which is precisely the case we are interested in.

Observe also that the close connection between our discrete framework and the framework for problem (4) is crucial for obtaining the convergence rate.

Theorem 5. Take $r$ and $\rho$ positive functions on $\mathbb{R}$, and assume that $\rho(x) \geq C>0$ on $\mathbb{R}$, with $C$ a constant. Assume also that (1)-(3) in Assumption 1 are satisfied. Let $u(t) \in W^{2,2}, v(t) \in W^{3,2}$, for all $t \in[0, T]$. Then there exists a constant $N$ independent of $h$ such that

1. $\sum_{x \in Z_{h}} r^{2}(x)\left|\frac{\partial}{\partial x} u(t, x)-\partial^{+} u(t, x)\right|^{2} \rho^{2}(x) h \leq h^{2} N\|u(t)\|_{2,2}^{2}$ 
2. $\sum_{x \in Z_{h}} r^{2}(x)\left|\frac{\partial^{2}}{\partial x^{2}} v(t, x)-\partial^{-} \partial^{+} v(t, x)\right|^{2} \rho^{4}(x) h \leq h^{2} N\|v(t)\|_{3,2}^{2}$,

for all $t \in[0, T]$.

Remark 4. Under the hypotheses of the above theorem, the function $u(t)$ (the function $v(t)$ ) has a modification in $x$ which is continuously differentiable in $x$ up to the order 1 (up to the order 2), for every $t \in[0, T]$. Also, the partial derivatives in $x$ up to the order 2 (up to the order 3 ) equal the weak derivatives a.e., for every $t \in[0, T]$.

These assertions can be proved by Sobolev's embedding of $W^{m, 2}(B)$ into $C^{n}(\bar{B})$, with $B$ a ball in $\mathbb{R}$, if $m>\frac{1}{2}+n$, and by Morrey's inequality (see, e.g., $[7,16]$ ). These modifications are considered in the proof of the theorem.

Remark 5. When particularising the hypotheses of the corresponding multidimensional result in [10] to the case of one spatial dimension, we obtain as assumptions that $u(t) \in W^{3,2}$ and $v(t) \in W^{4,2}$, for all $t \in[0, T]$, what is stronger than assumed in Theorem 5 .

In fact, in [10], the result is obtained making use of a Sobolev's embedding, while this is avoided in the proof of Theorem 5, by exploring the particular geometry of $\mathbb{R}$ which allows us to consider weaker assumtpions.

Proof. (Theorem 5) We prove (1). Observe that the forward difference quotient can be written

$$
\partial^{+} u(t, x)=h^{-1}(u(t, x+h)-u(t, x))=\int_{0}^{1} \frac{\partial}{\partial x} u(t, x+h q) d q .
$$

Thus

$$
\begin{aligned}
\left(\frac{\partial}{\partial x}-\partial^{+}\right) u(t, x) & =\int_{0}^{1}\left(\frac{\partial}{\partial x} u(t, x)-\frac{\partial}{\partial x} u(t, x+h q)\right) d q \\
& =h \int_{0}^{1} \int_{0}^{1} q \frac{\partial^{2}}{\partial x^{2}} u(t, x+h q s) d s d q .
\end{aligned}
$$

From (9), using Jensen's inequality, we obtain 


$$
\begin{aligned}
\left|\left(\frac{\partial}{\partial x}-\partial^{+}\right) u(t, x)\right|^{2} & \leq h^{2} \int_{0}^{1} \int_{0}^{1} q^{2}\left|\frac{\partial^{2}}{\partial x^{2}} u(t, x+h q s)\right|^{2} d s d q \\
& =h \int_{0}^{1} \int_{0}^{h q} q\left|\frac{\partial^{2}}{\partial x^{2}} u(t, x+v)\right|^{2} d v d q \\
& \leq h \int_{0}^{1} q d q \int_{0}^{h}\left|\frac{\partial^{2}}{\partial x^{2}} u(t, x+v)\right|^{2} d v \\
& =\frac{h}{2} \int_{0}^{h}\left|\frac{\partial^{2}}{\partial x^{2}} u(t, x+v)\right|^{2} d v \\
& =\frac{h}{2} \int_{x}^{x+h}\left|\frac{\partial^{2}}{\partial z^{2}} u(t, z)\right|^{2} d z .
\end{aligned}
$$

Observe also that from (10), using (3) in Assumption 1 we have, for any $\theta \in(0,1)$

$$
\begin{aligned}
r^{2}(x) \mid & \left.\left(\frac{\partial}{\partial x}-\partial^{+}\right) u(t, x)\right|^{2} \rho^{2}(x) \\
& \leq h N r^{2}(x+\theta h) \rho^{2}(x+\theta h) \int_{x}^{x+h}\left|\frac{\partial^{2}}{\partial z^{2}} u(t, z)\right|^{2} d z .
\end{aligned}
$$

As, by the mean value theorem for integration, for some $\theta \in(0,1)$,

$$
\begin{aligned}
r^{2}(x+\theta h) \rho^{2}(x+\theta h) & \int_{x}^{x+h}\left|\frac{\partial^{2}}{\partial z^{2}} u(t, z)\right|^{2} d z \\
& =\int_{x}^{x+h} r^{2}(z)\left|\frac{\partial^{2}}{\partial z^{2}} u(t, z)\right|^{2} \rho^{2}(z) d z,
\end{aligned}
$$

from (11) and (12), using Hölder inequality, we obtain

$$
\begin{aligned}
r^{2}(x) \mid & \left.\left(\frac{\partial}{\partial x}-\partial^{+}\right) u(t, x)\right|^{2} \rho^{2}(x) \\
& \leq h N \int_{x}^{x+h} r^{2}(z)\left|\frac{\partial^{2}}{\partial z^{2}} u(t, z)\right|^{2} \rho^{4}(z) d z \cdot \sup _{z \in[x, x+h]}\left|\rho^{-2}(z)\right| \\
& \leq h N \int_{x}^{x+h} r^{2}(z)\left|\frac{\partial^{2}}{\partial z^{2}} u(t, z)\right|^{2} \rho^{4}(z) d z
\end{aligned}
$$

owing to the hypotheses on the weights $\rho$. 
Finally, summing up (13) over $Z_{h}$, we get

$$
\sum_{x \in Z_{h}} r^{2}(x)\left|\left(\frac{\partial}{\partial x}-\partial^{+}\right) u(t, x)\right|^{2} \rho^{2}(x) h \leq h^{2} N\|u(t)\|_{2,2}^{2},
$$

with $N$ a constant independent of $h$, and (1) is proved.

We now prove (2). By writing the forward and backward difference quotients

and

$$
\partial^{+} v(t, x)=h^{-1}(v(t, x+h)-v(t, x))=\int_{0}^{1} \frac{\partial}{\partial x} v(t, x+h q) d q
$$

$$
\partial^{-} v(t, x)=h^{-1}(v(t, x)-v(t, x-h))=\int_{0}^{1} \frac{\partial}{\partial x} v(t, x-h s) d s,
$$

respectively, we have for the second-order difference quotient

$$
\begin{aligned}
\partial^{-} \partial^{+} v(t, x) & =\partial^{-} \int_{0}^{1} \frac{\partial}{\partial x} v(t, x+h q) d q \\
& =\int_{0}^{1}\left(\frac{\partial}{\partial x} \int_{0}^{1} \frac{\partial}{\partial x} v(t, x+h q-h s) d q\right) d s \\
& =\int_{0}^{1} \int_{0}^{1} \frac{\partial^{2}}{\partial x^{2}} v(t, x+h(q-s)) d s d q
\end{aligned}
$$

Thus,

$$
\begin{aligned}
& \left(\frac{\partial^{2}}{\partial x^{2}}-\partial^{-} \partial^{+}\right) v(t, x) \\
& =\int_{0}^{1} \int_{0}^{1}\left(\frac{\partial^{2}}{\partial x^{2}}(t, x)-\frac{\partial^{2}}{\partial x^{2}} v(t, x+h(q-s))\right) d s d q \\
& =h \int_{0}^{1} \int_{0}^{1} \int_{0}^{1}(q-s) \frac{\partial^{3}}{\partial x^{3}} v(t, x+h v(q-s)) d v d s d q .
\end{aligned}
$$

From (14), by Jensen's inequality,

$$
\begin{aligned}
& \left|\left(\frac{\partial^{2}}{\partial x^{2}}-\partial^{-} \partial^{+}\right) v(t, x)\right|^{2} \\
& \leq h^{2} \int_{0}^{1} \int_{0}^{1} \int_{0}^{1}|q-s|^{2}\left|\frac{\partial^{3}}{\partial x^{3}} v(t, x+h v(q-s))\right|^{2} d v d s d q \\
& =h \int_{0}^{1} \int_{0}^{1} \int_{0}^{h(q-s)}(q-s)\left|\frac{\partial^{3}}{\partial x^{3}} v(t, x+w)\right|^{2} d w d s d q
\end{aligned}
$$




$$
\begin{aligned}
& \leq h \int_{0}^{1} \int_{0}^{1}|q-s| d s d q \int_{0}^{h}\left|\frac{\partial^{3}}{\partial x^{3}} v(t, x+w)\right|^{2} d w \\
& \leq h \int_{0}^{h}\left|\frac{\partial^{3}}{\partial x^{3}} v(t, x+w)\right|^{2} d w=h \int_{x}^{x+h}\left|\frac{\partial^{3}}{\partial z^{3}} v(t, z)\right|^{2} d z,
\end{aligned}
$$

and, following the same steps as in the proof of (1), we finally obtain

$$
\sum_{x \in Z_{h}} r^{2}(x)\left|\left(\frac{\partial^{2}}{\partial x^{2}}-\partial^{-} \partial^{+}\right) v(t, x)\right|^{2} \rho^{4}(x) h \leq h^{2} N\|v(t)\|_{3,2}^{2},
$$

with $N$ a constant independent of $h$, and (2) is proved.

Following the same steps as in the proof of the corresponding result in [10] for the multidimensional case, and having the stability and consistency properties of the approximation scheme into account (Theorems 4 and 5, respectively), we can prove the convergence of the discrete problem's solution to the solution of the PDE problem, and compute a rate of convergence. The accuracy of the scheme is of order 1 .

The result is obtained by imposing additional regularity on the exact solution of problem (4) so that Theorem 5 holds, but lesser than it is assumed in the corresponding result in [10] (see Remark 5).

Theorem 6. Let $r$ and $\rho$ be positive functions on $\mathbb{R}$, and assume that $\rho(x) \geq C$ on $\mathbb{R}$, with $C$ a positive constant. Assume that the hypotheses of Theorems 2 and 4 hold. Denote by $u$ the solution of problem (4) given by Theorem 2 and by $u_{h}$ the solution of problem (8) in Theorem 4. Assume also that $u \in L^{2}\left([0, T] ; W^{3,2}\right)$, and that (3) in Assumption 1 is satisfied. Then

$$
\begin{aligned}
& \sup _{0 \leq t \leq T}\left\|u(t)-u_{h}(t)\right\|_{0,2}^{2}+\int_{0}^{T}\left\|u(t)-u_{h}(t)\right\|_{1,2}^{2} d t \\
& \leq h^{2} N \int_{0}^{T}\|u(t)\|_{3,2}^{2} d t+N\left(\left\|g-g_{h}\right\|_{0,2}^{2}+\int_{0}^{T}\left\|f(t)-f_{h}(t)\right\|_{0,2}^{2} d t\right),
\end{aligned}
$$

with $N$ a constant independent of $h$.

Remark 6. Under the hypotheses of the Theorem 6, there exist modifications in $x$ that ensure the continuity of $f(t)$ and $g$ in $x$, for every $t \in[0, T]$ (see Remark 4). These modifications will be considered in the proof of the theorem. 
Proof. (Theorem 6) From (4) and (8), the function $u-u_{h}$ satisfies the problem

$$
\left\{\begin{array}{l}
\left(u-u_{h}\right)_{t}=L_{h}\left(u-u_{h}\right)+\left(L-L_{h}\right) u+\left(f-f_{h}\right) \text { in } Q(h) \\
\left(u-u_{h}\right)(0, x)=\left(g-g_{h}\right)(x) \text { in } Z_{h} .
\end{array}\right.
$$

By Remark 6, it follows that $f-f_{h} \in L^{2}\left([0, T] ; l^{0,2}\right)$ and $g-g_{h} \in l^{0,2}$. As for $\left(L-L_{h}\right) u$, observe that for $u(t) \in W^{3,2}$ for all $t \in[0, T]$,

$$
\begin{aligned}
& \sum_{x \in Z_{h}} r^{2}(x)\left|\left(L-L_{h}\right)(t) u(t)\right|^{2} h \\
& =\sum_{x \in Z_{h}} r^{2}(x) \mid a(t, x)\left(\frac{\partial^{2}}{\partial x^{2}}-\partial^{-} \partial^{+}\right) u(t, x) \\
& \quad+\left.b(t, x)\left(\frac{\partial}{\partial x}-\partial^{+}\right) u(t, x)\right|^{2} h \leq h^{2} N\|u(t)\|_{3,2}^{2}<\infty,
\end{aligned}
$$

due to (2) in Assumption 2 and to Theorem 5. Thus $\left(L-L_{h}\right)(t) u(t) \in l^{0,2}$, for every $t \in[0, T]$. Moreover, as it is assumed that $u \in L^{2}\left([0, T] ; W^{3,2}\right)$, we derive immediately $\left(L-L_{h}\right) u \in L^{2}\left([0, T] ; l^{0,2}\right)$.

As we showed that problem (15) satisfies the hypotheses of Theorem 4, the following estimate holds

$$
\begin{aligned}
\sup _{0 \leq t \leq T}\left\|u(t)-u_{h}(t)\right\|_{0,2}^{2} & +\int_{0}^{T}\left\|u(t)-u_{h}(t)\right\|_{1,2}^{2} d t \\
\leq N & \left(\left\|g-g_{h}\right\|_{0,2}^{2}+\int_{0}^{T}\left\|f(t)-f_{h}(t)\right\|_{0,2}^{2} d t\right. \\
& \left.\quad+\int_{0}^{T}\left\|\left(L-L_{h}\right)(t) u(t)\right\|_{0,2}^{2} d t\right) .
\end{aligned}
$$

Again from (2) in Assumption 2 and taking Theorem 5 into account, the result follows.

As an immediate consequence of Theorem 6 we obtain the following result.

Corollary 7. Suppose that the hypotheses of Theorem 6 hold. Denote by $u$ the solution of (4) in Theorem 2 and by $u_{h}$ the solution of (8) given by Theorem 4. If there is a constant $N$ independent of the discretisation step $h$ such that 


$$
\begin{aligned}
\left\|g-g_{h}\right\|_{0,2}^{2}+ & \int_{0}^{T}\left\|f(t)-f_{h}(t)\right\|_{0,2}^{2} d t \\
& \leq h^{2} N\left(\|g\|_{0,2}^{2}+\int_{0}^{T}\|f(t)\|_{-1,2}^{2} d t\right),
\end{aligned}
$$

then

$$
\begin{aligned}
\sup _{0 \leq t \leq T} & \left\|u(t)-u_{h}(t)\right\|_{0,2}^{2}+\int_{0}^{T}\left\|u(t)-u_{h}(t)\right\|_{1,2}^{2} d t \\
& \leq h^{2} N\left(\int_{0}^{T}\|u(t)\|_{3,2}^{2} d t+\|g\|_{0,2}^{2}+\int_{0}^{T}\|f(t)\|_{-1,2}^{2} d t\right) .
\end{aligned}
$$

\section{An example}

In this section we illustrate the above approximation study by considering an example.

In problem (4), we specify the PDE coefficients

$$
a(t, x)=(1+t)\left(1+x^{2}\right), \quad b(t, x)=2 t x, \quad \text { and } \quad c(t, x)=\sin (t x),
$$

and the free data

$$
f(t, x)=t\left(1+x^{3}\right), \quad \text { and } \quad g(x)=\left(1+x^{3}\right) .
$$

Then, problem (4) reads

$$
u_{t}=L u+t\left(1+x^{3}\right) \text { in } Q, \quad u(0, x)=\left(1+x^{3}\right) \text { on } \mathbb{R},
$$

where

$$
L=(1+t)\left(1+x^{2}\right) \frac{\partial^{2}}{\partial x^{2}}+2 t x \frac{\partial}{\partial x}+\sin (t x) .
$$

Consider also the weight functions

$$
r(x)=\left(1+x^{2}\right)^{\beta} \text {, with } \beta<-11 / 4, \quad \text { and } \quad \rho(x)=\left(1+x^{2}\right)^{1 / 2} .
$$

Take $m=2$, and consider the functional setting in Section 2 as underlying the study of problem (16). It can be easily checked that the hypotheses of Theorem 2 are satisfied:

- the weights $r$, and $\rho$ satisfy (1) - (2) in Assumption 1 (with $m=3$ as required in Theorem 2); 
- the coefficients $a, b$, and $c$ satisfy (1) - (2) in Assumption 2;

- the free data $f$, and $g$ satisfy (3) in Assumption 2.

Thus Theorem 2 ensures that there exists a unique generalised solution $u$ of the particular PDE problem (16) such that

and

$$
u \in C\left([0, T] ; W^{2,2}\right) \cap L^{2}\left([0, T] ; W^{3,2}\right),
$$

$$
\sup _{0 \leq t \leq T}\|u(t)\|_{2,2}^{2}+\int_{0}^{T}\|u(t)\|_{3,2}^{2} d t \leq N\left(\|g\|_{2,2}^{2}+\int_{0}^{T}\|f(t)\|_{1,2}^{2} d t\right),
$$

with $N$ a constant.

We now discretise problem (16) on the $h$-grid $Z_{h}$ on $\mathbb{R}$ defined by (5).

For $x \in Z_{h}$ and $t \in[0, T]$, define

$$
f_{h}(t, x):=f(t, x)=t\left(1+x^{3}\right), \quad \text { and } \quad g_{h}(x):=g(x)=\left(1+x^{3}\right),
$$

discrete versions of $f$ and $g$, respectively. We then obtain the family of ordinary differential equations in the time variable $t$

$$
u_{t}(t, x)=L_{h} u(t, x)+t\left(1+x^{3}\right) \text { in } Q(h),
$$

with $Q(h)=[0, T] \times Z_{h}$, and satisfying

$$
u(0, x)=\left(1+x^{3}\right) \text { in } Z_{h},
$$

where the operator $L_{h}$ is defined by

$$
L_{h}(t, x):=a(t, x) \partial^{+} \partial^{-}+b(t, x) \partial^{+}+c(t, x),
$$

with $\partial^{+}$and $\partial^{-}$the forward and backward difference quotient operators in space defined, respectively, by (6) and (7).

Let us consider problem (19) - (20) under the discrete functional setting in Section 2. It is clear that:

- $f_{h} \in L^{2}\left([0, T] ; l^{0,2}\right) \quad$ and $\quad g_{h} \in l^{0,2}$,

and so (1) - (2) in Assumption 3 are satisfied. Thus the hypotheses of Theorem 4 are satisfied, and the result ensures that there exists a unique generalised solution $u_{h}$ of the particular problem (19) - (20).

For the convergence, notice that

- $\rho(x)=\left(1+x^{2}\right)^{1 / 2} \geq 1$ on $\mathbb{R}$;

- $u \in L^{2}\left([0, T] ; W^{3,2}\right)$, by $(17)$. 
Consequently, the convergence result Theorem 6 holds. Moreover, as

$$
\left\|g-g_{h}\right\|_{0,2}^{2}+\int_{0}^{T}\left\|f(t)-f_{h}(t)\right\|_{0,2}^{2} d t=0
$$

due to the chosen discretisation of $f$ and $g$, owing to (18) the rate of convergence of the generalised solution of problem $(19)-(20)$ to the generalised solution of problem (16) in Theorem 6 writes

$$
\begin{aligned}
\sup _{0 \leq t \leq T}\left\|u(t)-u_{h}(t)\right\|_{0,2}^{2} & +\int_{0}^{T}\left\|u(t)-u_{h}(t)\right\|_{1,2}^{2} d t \\
& \leq h^{2} N\left(\|g\|_{2,2}^{2}+\int_{0}^{T}\|f(t)\|_{1,2}^{2} d t\right)
\end{aligned}
$$

with $N$ a constant independent of $h$.

\section{Final remarks}

We investigated the finite-difference spatial approximation of the Cauchy problem for linear parabolic PDEs of second order with nondivergent operator and unbounded time- and space-dependent coefficients. By concentrating on the case of one spatial dimension, we could exploit specific geometric properties of $\mathbb{R}$ and deduce consistency and convergence results sharper than those obtained in [10] for the more general multidimensional case. An estimate for the rate of convergence was given.

The approximation was pursued in the framework of the variational approach. The integrability issues raised by the unboundedness in space of the partial differential operator coefficients, and the possible spacial growth of both the free term $f$ and the initial data $g$, were handled by considering a suitable class of weighted Sobolev spaces, and its zero and first-order discrete versions.

Our work presents two obvious limitations: the assumption that the PDE does not degenerate, and also low accuracy of the approximation we produced, which is not good enough for practical purposes.

In this connection, possible further research directions include: (i) the approach of the corresponding degenerate case (for this, the results obtained in $[12,13]$ and also the work [1] will play an important role), and (ii) the use of splitting-up methods (see [14]), following Richardson's idea to accelerate numerical schemes.

Acknowledgements. The authors were supported by Portuguese funds through FCT/MCTES: the first two (FFG and MRG) within Project CEMAPRE- 
UID/MULTI/00491/2019, and the third (EM) within Project UID/MAT/00013/ 2013.

\section{References}

[1] Y. Achdou, O. Pironneau, Computational Methods for Option Pricing, Frontiers in Applied Mathematics, SIAM, USA (2005).

[2] G. Barles, Ch. Daher and M. Romano, Convergence of numerical schemes for parabolic equations arising in Finance theory, Math. Models Methods Appl. Sci., 5 No 1 (1995), 125-143.

[3] M. Broadie and J. B. Detemple, Option pricing: valuation models and applications, Manag. Sci., 50, No 9 (2004), 1145-1177.

[4] J. Douglas Jr. and T.F. Russel, Numerical methods for convectiondominated diffusion problems based on combining the method of characteristics with finite element or finite difference procedures, SIAM J. Numer. Anal., 19, No 5 (1982), 871-885.

[5] M. Ehrhardt, Finite difference schemes on unbounded domains, In: Applications of Nonstandard Finite Difference Schemes (Vol. 2) (Ed. R.E. Mickens), World Scientific (2005), 343-384.

[6] M. Ehrhardt, Discrete transparent boundary conditions for Schrödingertype equations for non-compactly supported initial data, Appl. Numer. Math., 58, No 5 (2008), 660-673.

[7] L.C. Evans, Partial Differential Equations, Graduate Studies in Mathematics 19, Amer. Math. Soc., USA (1998).

[8] F.F. Gonçalves, Numerical approximation of partial differential equations arising in financial option pricing, Ph.D. Thesis, University of Edinburgh, UK (2007).

[9] F.F. Gonçalves, M.R. Grossinho and E. Morais, Discretisation of abstract linear evolution equations of parabolic type. Adv. Difference Equ., 2012, No 14 (2012), 1-29; doi: 10.1186/1687-1847-2012-14.

[10] F.F. Gonçalves and M.R. Grossinho, Spatial approximation of nondivergent type parabolic PDEs with unbounded coefficients related to finance, Abstr. Appl. Anal. (2014), Special Issue: Functional Differential and Difference Equations with Applications (2013); doi: 10.1155/2014/801059. 
[11] I. Gyöngy and N.V. Krylov, Stochastic partial differential equations with unbounded coefficients and applications I, Stochastics, 32 (1990), 53-91.

[12] I. Gyöngy and N.V. Krylov, First derivatives estimates for finite-difference schemes, Math. Comp., 78 (2009), 2019-2046.

[13] I. Gyöngy and N.V. Krylov, Higher order derivative estimates for finitedifference schemes for linear elliptic and parabolic equations, Methods Appl. Anal., 16, No 2 (2009), 187-216.

[14] I. Gyöngy and N.V. Krylov, Accelerated finite difference schemes for second order degenerate elliptic and parabolic problems in the whole space, Math. Comp., 80, No 275 (2011), 1431-1458.

[15] D. Lamberton and B. Lapeyre, Introduction to Stochastic Calculus Applied to Finance. Chapman and Hall, UK (1996).

[16] J.-L. Lions and E. Magenes, Problèmes aux Limites Non Homogènes et Applications, Vol. 1 (in French), Dunod, Gauthier-Villars, Paris, France (1968).

[17] P. Lötstedt, J. Persson, L. von Sydow and J. Tysk, Spacetime adaptive finite difference method for European multi-asset options, J. Comput. Math. Appl., 53, No 8 (2007), 1159-1180.

[18] E. Morais, Analytical and Numerical Methods for Some Problems Related to Financial Option Pricing, Ph.D. Thesis, University of Lisbon, Portugal (2013).

[19] D.M. Pooley, P.A. Forsyth and K.R. Vetzal, Numerical convergence properties of option pricing PDEs with uncertain volatility, IMA J. Numer. Anal., 23 (2003), 241-267.

[20] D.Y. Tangman, A. Gopaul and M. Bhuruth, Numerical pricing of options using high-order compact finite difference schemes, J. Comput. Appl. Math., 218 (2008), 270-280.

[21] V. Thomée, Finite difference methods for linear parabolic equations, In: Finite Difference Methods (Part 1) - Solution of Equations in $\mathbb{R}^{n}$ (Part 1), Handbook of Numerical Analysis, Vol. I (Ed. P.G. Ciarlet, J.L. Lions), North-Holland (1990), 3-196. 